OPEN ACCESS

Edited by:

Andrea P. Rossi,

Ca' Foncello Hospital, Italy

Reviewed by:

Marios Kyriazis,

National Gerontology Centre, Cyprus

Bo Jia,

Shanghai Ninth People's

Hospital, China

*Correspondence:

Xiaodong Sun

xdsun@sjtu.edu.cn

Minwen Zhou

zmw8008@163.com

tThese authors have contributed equally to this work and share first authorship

Specialty section:

This article was submitted to Geriatric Medicine,

a section of the journal

Frontiers in Medicine

Received: 16 September 2021 Accepted: 08 December 2021 Published: 24 December 2021

Citation:

Sun $M, B o Q, L u B$, Sun X and Zhou M (2021) The Association of Sleep Duration With Vision Impairment in Middle-Aged and Elderly Adults:

Evidence From the China Health and Retirement Longitudinal Study. Front. Med. 8:778117.

doi: 10.3389/fmed.2021.778117

\section{The Association of Sleep Duration With Vision Impairment in Middle-Aged and Elderly Adults: Evidence From the China Health and Retirement Longitudinal Study}

\author{
Mengsha Sun ${ }^{1 \dagger}$, Qiyu Bo ${ }^{2 \dagger}$, Bing Lü ${ }^{2}$, Xiaodong Sun ${ }^{2,3,4 *}$ and Minwen Zhou ${ }^{2,3,4 *}$ \\ 1 Department of Ophthalmology, Shanghai East Hospital, Tongji University School of Medicine, Shanghai, China, \\ ${ }^{2}$ Department of Ophthalmology, Shanghai General Hospital, Shanghai First People's Hospital, Shanghai Jiao Tong University \\ School of Medicine, Shanghai, China, ${ }^{3}$ National Clinical Research Center for Eye Diseases, Shanghai, China, ${ }^{4}$ Shanghai Key \\ Laboratory of Fundus Diseases, Shanghai, China
}

Objective: This study aims to investigate the association of sleep duration with vision impairment $(\mathrm{VI})$ in middle-aged and elderly adults.

Methods: This cross-sectional study used the data from the baseline survey of the China Health and Retirement Longitudinal Study (CHARLS) 2011-2012, a national survey of adults aged 45 years or older. Weighted multilevel logistic regression models were used to evaluate the association between self-reported sleep duration and VI.

Results: Of the 13,959 survey respondents, a total of 4,776 (34.2\%) reported VI. The prevalence of short ( $\leq 6 \mathrm{~h} / \mathrm{night}$ ) and long ( $>8 \mathrm{~h} / \mathrm{night}$ ) sleep durations was higher among respondents with $\mathrm{VI}$ than those without $\mathrm{VI}(P<0.001)$. Multilevel logistic regression models showed that compared with a sleep duration of 6-8 h/night, a sleep duration of $\leq 6 \mathrm{~h} /$ night was associated with a 1.45-fold [95\% confidence interval $(\mathrm{Cl})=1.34-1.56$ ] higher VI risk, and a sleep duration of $>8 \mathrm{~h} / \mathrm{hight}$ was associated with a 1.18-fold $195 \% \mathrm{Cl}$ $=1.03-1.34$ ) higher VI risk, after adjusting for sociodemographic data, lifestyle factors, and health conditions. Vision impairment was associated with short sleep duration in respondents from all age or gender categories. However, VI was associated with long sleep duration in respondents from the elderly or female categories. The association between $\mathrm{VI}$ and long sleep duration disappeared in respondents of middle-aged or male categories.

Conclusions: The potential impact of sleep on the risk of visual functions requires further attention. A more comprehensive and integrated health care and rehabilitation system covering vision and sleep is also needed.

Keywords: vision impairment, sleep duration, association, adults, China 


\section{INTRODUCTION}

Vision impairment (VI) is a major global public health problem. According to a published report in Lancet Global Health, the number of people affected by VI has increased substantially, a situation attributable to population growth and aging (1). The combination of cataract and uncorrected refractive error contributed to $55 \%$ of the blindness and $77 \%$ of the $\mathrm{VI}$ in adults aged 50 years and older in 2015 (1). In China, the prevalence of low bilateral vision was $5.1 \%$, and the prevalence of blindness was $1.0 \%$, based on the World Health Organization (WHO) best corrected visual acuity (BCVA) criteria in the Taizhou Eye Study (2). Further analysis revealed that VI was associated with an increased rate of cognitive impairment and depression and a lowered quality of life (3-5). A few studies based on the US population have concluded that older adults with VI also experienced increased sleep disturbance $(6,7)$.

In terms of VI, sleep is one of the aspects of functioning that may be underestimated because attention is focused on establishing visual functions. Sleep is vital for optimal physical and mental health, and growing evidence now supports an association of both short- and long-duration sleep with adverse health outcomes (8-10). Several epidemiologic studies have reported an increased risk of all-cause mortality, as well as stroke and cardiovascular disease mortality, among those with self-reported short or long sleep durations (11-13). Recent findings have also uncovered differences in sleep health based on racial/ethnic and socioeconomic position differences. The underlying causes of disparities in sleep health are only beginning to emerge (14).

Recent studies have reported a statistically significant association between sleep disorders and ocular diseases, such as glaucoma, optic nerve dysfunction, and dry eye syndrome (1517). However, sleep data have not yet been considered an essential element in many epidemiologic studies of VI. To our knowledge, few large-scale population-based studies have examined the potential association between sleep duration and VI in an Asian population. The aim of the present study was to evaluate the associations between sleep duration and the prevalence of $\mathrm{VI}$ in a nationally representative survey of the Chinese adult population aged 45 years or older, using data from the 2011 baseline survey of the China Health and Retirement Longitudinal Study (CHARLS).

\section{MATERIALS AND METHODS}

\section{Data and Study Sampling}

The CHARLS is a nationally representative survey of Chinese adults aged 45 years or older and their spouses. The national baseline survey for CHARLS was conducted between June 2011 and March 2012 and included 17,708 respondents in 150 counties or districts and 450 villages or urban communities throughout China (18). The CHARLS protocol followed the tenets of the Declaration of Helsinki (19) and received ethical approval from the institutional review board of Peking

Abbreviations: VI, visual impairment; OR, odds ratio; CI, confidence interval; CHARLS, China Health and Retirement Longitudinal Study.
University (18). Informed consent was obtained from all participants when CHARLS was administered, consistent with the Strengthening the Reporting of Observational Studies in Epidemiology (STROBE) reporting guidelines (20).

\section{Vision Impairment}

Vision consisted of both distance vision and near vision. Distance vision was assessed by asking the participants: "How good is your eyesight for seeing things at a distance, like recognizing a friend from across the street (with glasses or corrective lenses if you wear them)?" Near vision was assessed by asking the participants: "How good is your eyesight for seeing things up close, like reading ordinary newspaper print (with glasses or corrective lenses if you wear them)?" Responses to these two questions were divided into a five-point scale (excellent, very good, good, fair, and poor). A response of "poor" in distance or near vision was categorized as VI, while a response of "fair" or above in distance and near vision was categorized as no VI (21).

\section{Sleep Duration}

Sleep duration was assessed by asking participants the following question: "During the past month, how many hours of actual sleep did you get at night (average hours for one night)? (This may be shorter than the number of hours you spend in bed)." The participant responses for sleep duration were categorized into three groups: (1) those reporting short sleep duration $(\leq 6 \mathrm{~h})$, (2) those reporting long sleep duration $(>8 \mathrm{~h})$, and (3) those reporting $6-8 \mathrm{~h}$ of sleep (used as the reference group) $(22,23)$.

\section{Covariates}

Several potential confounding variables were taken into account, including participants' sociodemographic data, lifestyle factors, and health conditions. The sociodemographic data and lifestyle factors included in the analysis were as follows: age, sex (male or female), marital status (married or partnered vs. otherwise), smoking status (yes or no), drinking status (more than once a month, less than once a month, or none), and educational attainment (less than elementary school, elementary school, middle school, or high school or above).

Health conditions included in the analysis were hypertension $(24,25)$, dyslipidemia (26), diabetes (27), and chronic kidney disease (28-30), assessed by a combination of physical examination and self-report according to reference definition. In CHARLS, blood samples were collected and blood pressure was measured using a standard protocol by medically trained staff from the China Center for Disease Control and Prevention (31).

\section{Statistical Analysis}

Because CHARLS has a complex sampling design, sampling weights were considered in the analyses. Continuous variables were presented using mean $\pm \mathrm{SD}$. Categorical variables were presented using numbers. Characteristics of the study sample were compared using the $\chi^{2}$-test for categorical variables and an independent-sample $t$-test for continuous variables. The subjects were divided into two groups according to gender or the median age: middle age and elderly. The prevalence of VI was calculated based on the complex survey design and non-response rate. 
The PROC SURVEYFREQ procedure was used to calculate the overall age and gender-specific prevalence of VI using the inverse probability weighting method. Weighted multilevel logistic regression models were used to evaluate the odds ratio $(\mathrm{OR})$ at a $95 \%$ confidence interval (CI). They modeled the association between self-reported sleep duration and VI, adjusting for sociodemographic data (age, sex), lifestyle factors (marital status, smoking status, drinking status, and education), and health conditions (hypertension, dyslipidemia, diabetes, and chronic kidney disease). Statistical analysis was carried out using the Statistical Analysis System (SAS) (version 9.4, SAS Institute, Cary, NC, USA) for Windows. All reported $P$-values were twotailed, and values $<0.05$ were considered statistically significant.

\section{RESULTS}

After filtering, a total of 13,959 participants were deemed eligible for the current study. In total, 6,574 males and 7,385 females were included in the final analysis. The mean age was 59.97 (9.84) years. The characteristics of the included subjects in the CHARLS 2011-2012 are shown in Table 1. Of the study subjects, 4,776 reported VI, with a higher proportion of females than males reporting VI. Those who reported VI were older, less educated, and more likely to be unmarried or unpartnered. The subjects with VI were also more likely to have smoking history, hypertension, diabetes, and chronic kidney disease. Interestingly, sleep durations showed some relationships with VI. The prevalence of short ( $\leq 6 \mathrm{~h} /$ night) and long ( $>8 \mathrm{~h} /$ night) sleep durations was higher among respondents with VI than those without VI $(P<0.001)$.

The weighted VI prevalences in respondents, by age and sex, based on sleep durations are shown in Table 2. The prevalence of VI among the study respondents was $35.83 \%$. More specifically, the prevalence of VI was higher among respondents with short $(40.78 \%)$ or long $(35.33 \%)$ sleep durations than those with sleep durations of 6-8 h (26.10\%).

By age, the VI prevalence was higher in elderly respondents (40.98\%) than in middle-aged respondents (31.08\%). Separately, the VI prevalence was higher in elderly respondents with short $(42.97 \%)$ or long $(40.19 \%)$ sleep durations than with sleep durations of $6-8 \mathrm{~h}(31.68 \%)$. The VI prevalence was also higher in middle-aged respondents with short (35.78\%) or long (25.80\%) sleep durations than with sleep durations of 6-8 h/night (23.60\%).

The VI prevalence was also higher in female respondents $(38.47 \%)$ than in male respondents $(32.89 \%)$. In the female participants, the VI prevalence was $45.84,30.26$, and $39.65 \%$ for subjects with sleep durations of $\leq 6,6-8$, and $>8 \mathrm{~h} / \mathrm{night}$, respectively. In the male participants, the equivalent VI prevalence was $35.61,26.88$, and $30.82 \%$, respectively. Overall, the VI prevalence was higher among respondents with short or long sleep durations than with normal sleep duration in all age and gender categories.

The associations between sleep duration and VI determined using multilevel logistic regression models are shown in Table 3. These associations had variations after covariate adjustments
TABLE 1 | Demographic characteristics of middle-aged and elderly Chinese with and without suffering vision impairment (CHARLS 2011-2012).

\begin{tabular}{|c|c|c|c|}
\hline Variables & $\begin{array}{l}\text { Vision } \\
\text { impairment }\end{array}$ & $\begin{array}{l}\text { No vision } \\
\text { impairment }\end{array}$ & $P$ \\
\hline Total & 4,776 & 9,183 & \\
\hline Age, year & $60.96 \pm 9.87$ & $59.09 \pm 9.58$ & $<0.001$ \\
\hline Sex & & & $<0.001$ \\
\hline Male & 1,980 & 4,594 & \\
\hline Female & 2,796 & 4,589 & \\
\hline Marital status & & & $<0.001$ \\
\hline Married or partnered & 4,055 & 8,191 & \\
\hline Otherwise & 721 & 992 & \\
\hline Smoking status & & & 0.002 \\
\hline Yes & 1,760 & 3,633 & \\
\hline No & 3,016 & 5,550 & \\
\hline Drinking status & & & 0.126 \\
\hline $\begin{array}{l}\text { Drink more than once a } \\
\text { month }\end{array}$ & 1,084 & 2,376 & \\
\hline $\begin{array}{l}\text { Drink but less than once } \\
\text { a month }\end{array}$ & 313 & 765 & \\
\hline None & 3,379 & 6,042 & \\
\hline Education & & & $<0.001$ \\
\hline $\begin{array}{l}\text { Less than elementary } \\
\text { school }\end{array}$ & 3,591 & 5,697 & \\
\hline Elementary school & 777 & 2,116 & \\
\hline Middle school & 256 & 813 & \\
\hline High school or above & 152 & 557 & \\
\hline Hypertension & & & $<0.001$ \\
\hline Yes & 1,994 & 3,339 & \\
\hline No & 2,782 & 5,844 & \\
\hline Dyslipidemia & & & 0.060 \\
\hline Yes & 1,624 & 2,977 & \\
\hline No & 3,152 & 6,206 & \\
\hline Diabetes & & & $<0.001$ \\
\hline Yes & 727 & 1,115 & \\
\hline No & 4,049 & 8,068 & \\
\hline $\begin{array}{l}\text { Chronic kidney } \\
\text { disease }\end{array}$ & & & $<0.001$ \\
\hline Yes & 407 & 495 & \\
\hline No & 4,369 & 8,688 & \\
\hline Sleep duration & & & $<0.001$ \\
\hline$\leq 6 \mathrm{~h}$ & 2,718 & 4,252 & \\
\hline $6-8 \mathrm{~h}$ & 1,646 & 4,138 & \\
\hline$>8 \mathrm{~h}$ & 412 & 793 & \\
\hline
\end{tabular}

for age, sex, marital status, smoking status, drinking status, education, hypertension, dyslipidemia, diabetes, and chronic kidney disease. Even after adjusting for these factors, a sleep duration of $\leq 6 \mathrm{~h} /$ night was associated with a 1.45 -fold (95\% CI $=1.34-1.56$ ) higher VI risk, while a sleep duration of $>8 \mathrm{~h} / \mathrm{night}$ was associated with a 1.18 -fold $(95 \% \mathrm{CI}=1.03-1.34)$ higher VI risk compared with 6-8 h/night of sleep.

A stratified analysis of age categories revealed that short $(\mathrm{OR}=1.44,95 \% \mathrm{CI}: 1.29-1.60)$ and long $(\mathrm{OR}=1.30,95 \%$ 
TABLE 2 | Vision impairment prevalence in middle-aged and elderly Chinese according to sleep durations (CHARLS 2011-2012).

\begin{tabular}{|c|c|c|c|c|c|}
\hline & \multirow[b]{2}{*}{ Overall } & \multicolumn{3}{|c|}{ Sleep duration groups (h/night) } & \multirow[t]{2}{*}{$\boldsymbol{P}$} \\
\hline & & $\leq 6 \mathrm{~h}$ & $6-8 \mathrm{~h}$ & $>8 \mathrm{~h}$ & \\
\hline All & $n=13,959$ & $n=6,970$ & $n=5,784$ & $n=1,205$ & \\
\hline Vision impairment, $n$ (\%) & $4,776(35.83)$ & $2,718(40.78)$ & $1,646(26.10)$ & $412(35.33)$ & $<0.001$ \\
\hline \multicolumn{6}{|l|}{ Age } \\
\hline$\geq 45$ and $\leq 58$ years & $n=7,239$ & $n=3,315$ & $n=3,320$ & $n=604$ & \\
\hline Vision impairment, $n$ (\%) & $2,101(31.08)$ & $1,125(35.78)$ & $813(23.60)$ & $163(25.80)$ & $<0.001$ \\
\hline$>58$ years & $n=6,720$ & $n=3,655$ & $n=2,464$ & $n=601$ & \\
\hline Vision impairment, $n$ (\%) & $2,675(40.98)$ & $1,593(42.97)$ & $833(31.68)$ & $249(40.19)$ & $<0.001$ \\
\hline \multicolumn{6}{|l|}{ Sex } \\
\hline Male & $n=6,384$ & $n=3,172$ & $n=2,849$ & $n=553$ & \\
\hline Vision impairment, $n$ (\%) & $1,980(32.89)$ & $1,097(35.61)$ & $721(26.88)$ & $162(30.82)$ & $<0.001$ \\
\hline Female & $n=7,385$ & $n=3798$ & $n=2,935$ & $n=652$ & \\
\hline Vision impairment, $n$ (\%) & $2,796(38.47)$ & $1,621(45.84)$ & $925(30.26)$ & $250(39.65)$ & $<0.001$ \\
\hline
\end{tabular}

CI: $1.08-1.57)$ sleep durations were associated with VI in elderly respondents. However, in middle-aged respondents, an association was only detected between short sleep duration and $\mathrm{VI}(\mathrm{OR}=1.45,95 \% \mathrm{CI}: 1.30-1.62)$. The association between long sleep duration and VI $(\mathrm{OR}=1.11,95 \% \mathrm{CI}=0.91-1.35)$ was absent among the middle-aged respondents.

A stratified analysis of gender categories revealed that short $(\mathrm{OR}=1.45,95 \% \mathrm{CI}: 1.30-1.60)$ and long $(\mathrm{OR}=1.24,95 \%$ CI: 1.03-1.48) sleep durations were associated with VI in female respondents. However, male respondents showed only the association between short sleep duration and VI $(\mathrm{OR}=1.45$, 95\% CI: 1.29-1.62). The association between long sleep duration and VI $(\mathrm{OR}=1.11,95 \% \mathrm{CI}=0.90-1.36)$ was absent among the male respondents.

\section{DISCUSSION}

This study examined the association between sleep duration and VI in a nationally representative sample of older adults in China. The use of large-scale population-based data in this study revealed several findings. First, self-reported VI was highly prevalent among middle-aged and elderly adults in China. Second, short ( $\leq 6 \mathrm{~h} /$ night) and long $(>8 \mathrm{~h} /$ night) sleep durations were significantly associated with VI risks after adjusting for internal and external factors. Our findings suggest that both short and long sleep durations could be predictors of VI.

The prevalence of VI in China was higher than that reported in the US or Asia (32). The rapidly aging Chinese population may account for this higher rate of VI. Chen et al. (33) reported that cataracts were the leading cause of bilateral or monocular VI among adults 50 years and older in the Binhu District of Wuxi City, China, followed by high myopic macular degeneration, agerelated macular degeneration, and eye loss/atrophy. Bourne et al. (34) reported that the leading causes worldwide were cataracts for blindness and uncorrected refractive error for moderate and severe VI. Moreover, more women than men were blind or showed moderate and severe VI due to cataracts and macular degeneration worldwide (34). Our results also revealed a higher prevalence of VI in women than in men, in agreement with previous studies. Therefore, more targeted efforts are required to prevent low vision and blindness in high-risk groups.

The mechanism that regulates sleep duration has not been fully explained. A short sleep duration may be attributed to difficulty in falling asleep, sleep fragmentation, and early awakening. Long sleep duration may occur due to poor circadian entrainment, compensation for fragmented sleep, and lower sleep efficiency $(10,35)$. Sleep deterioration and related VI may also result from several diseases (36). In turn, VI may further increase mood disturbances and even worsen sleep disorders. Further, respondents with VI tend to exhibit a shortened photoperiod and reduced circadian entrainment (8). The decline in or loss of light perception may lead to circadian misalignment, further contributing to abnormal sleep durations. Therefore, ophthalmologists must recognize the possibility of sleep disturbance in subjects with VI and participate in effective sleep management.

In addition to the above biological and clinical reasons, social factors should not be underestimated. Certain socioeconomic factors (including country of origin, acculturation, education, and annual income) have been found to be significantly associated with both short and long sleep duration $(37,38)$. These relationships persisted when controlling for overall health status. On the other hand, socioeconomic status is known to be an important determinant of VI (39). As such, socioeconomic status may play a role in the relationship between sleep duration and VI.

In particular, the results from the multilevel logistic regression models indicated a more significant potential for VI with short sleep duration than with long sleep duration. This suggests that persons who sleep for a short duration may require greater amounts of sleep and that worse sleep conditions pose a greater risk of VI. Additional studies examining the causal relationship between sleep durations and VI are needed.

To our knowledge, this is the first large-scale population-based study to explore the associations between self-reported sleep 
TABLE 3 | Associations between sleep duration and risk of vision impairment in middle-aged and elderly Chinese (CHARLS 2011-2012).

\begin{tabular}{|c|c|c|c|}
\hline & \multicolumn{3}{|c|}{ Sleep duration groups (h/night) } \\
\hline & $\leq 6 \mathrm{~h}$ & $6-8 \mathrm{~h}$ & $>8 \mathrm{~h}$ \\
\hline & OR (95\%Cl) & OR $(95 \% \mathrm{Cl})$ & OR $(95 \% \mathrm{Cl})$ \\
\hline All & $n=6,970$ & $n=5,784$ & $n=1,205$ \\
\hline Model 1 & $1.50(1.39-1.61)^{\star}$ & 1.00 (Ref.) & $1.23(1.07-1.40)^{\star}$ \\
\hline Model 2 & $1.47(1.36-1.58)^{\star}$ & 1.00 (Ref.) & $1.18(1.03-1.35)^{*}$ \\
\hline Model 3 & $1.45(1.34-1.56)^{\star}$ & 1.00 (Ref.) & $1.18(1.03-1.34)^{\star}$ \\
\hline \multicolumn{4}{|l|}{ Age } \\
\hline$\geq 45$ and $\leq 58$ years & $n=3,320$ & $n=3315$ & $n=604$ \\
\hline Model 1 & $1.50(1.35-1.68)^{\star}$ & 1.00 (Ref.) & $1.15(0.95-1.41)$ \\
\hline Model 2 & $1.47(1.32-1.64)^{\star}$ & 1.00 (Ref.) & $1.12(0.91-1.36)$ \\
\hline Model 3 & $1.45(1.30-1.62)^{\star}$ & 1.00 (Ref.) & $1.11(0.91-1.35)$ \\
\hline$>58$ years & $n=3,655$ & $n=2,464$ & $n=601$ \\
\hline Model 1 & $1.46(1.31-1.63)^{\star}$ & 1.00 (Ref.) & $1.35(1.12-1.62)^{\star}$ \\
\hline Model 2 & $1.45(1.30-1.62)^{\star}$ & 1.00 (Ref.) & $1.29(1.07-1.55)^{\star}$ \\
\hline Model 3 & $1.44(1.29-1.60)^{\star}$ & 1.00 (Ref.) & $1.30(1.08-1.57)^{\star}$ \\
\hline \multicolumn{4}{|l|}{ Sex } \\
\hline Male & $n=3,172$ & $n=2,849$ & $n=553$ \\
\hline Model 1 & $1.50(1.34-1.67)^{\star}$ & 1.00 (Ref.) & $1.15(0.94-1.41)$ \\
\hline Model 2 & $1.47(1.31-1.65)^{\star}$ & 1.00 (Ref.) & $1.11(0.90-1.36)$ \\
\hline Model 3 & $1.45(1.29-1.62)^{\star}$ & 1.00 (Ref.) & $1.11(0.90-1.36)$ \\
\hline Female & $n=3,798$ & $n=2,935$ & $n=652$ \\
\hline Model 1 & $1.49(1.35-1.65)^{\star}$ & 1.00 (Ref.) & $1.29(1.08-1.54)^{\star}$ \\
\hline Model 2 & $1.46(1.32-1.62)^{\star}$ & 1.00 (Ref.) & $1.23(1.03-1.48)$ * \\
\hline Model 3 & $1.45(1.30-1.60)^{\star}$ & 1.00 (Ref.) & $1.24(1.03-1.48)^{\star}$ \\
\hline
\end{tabular}

Model 1 adjusted for age and sex. Model 2 adjusted for covariates in model 1 plus marital status, smoking status, drinking status, and education. Model 3 adjusted for covariates in model 2 plus hypertension, dyslipidemia, diabetes, and chronic kidney disease. *Significant.

duration and VI among Chinese persons. Self-reported sleep duration and the prevalence of VI are known to reflect ethnic differences $(40,41)$. Therefore, investigation of the association between VI and sleep duration is essential among older adults in China. This type of research would be more convincing after adjusting for sociodemographic characteristics, lifestyle factors, and health conditions, as all these factors can affect the relationship between self-reported sleep duration and VI.

In fact, the potential impact between sleep and vision is underestimated. A health care and rehabilitation system that covers vision and sleep must be established and popularized. The system should take into account the possibility of VI in subjects with abnormal sleep duration as well as abnormal sleep duration in subjects with VI and engage in effective management accordingly. As for subjects with abnormal sleep duration, their vision function should be taken care of and examined regularly. As for subjects with VI, their sleep-wake cycles should be given close attention. Sleep medication, physical therapy, or psychotherapy treating sleep difficulties may be helpful in reducing the incidence of VI. Vision impairment medication or surgery may contribute to setting up circadian rhythm and improving abnormal sleep duration $(42,43)$.
The present study is not without limitations, which can be summarized as follows:

1. Due to the cross-sectional nature of the study, causality could not be established. Prospective cohort studies may need to clarify the causality between sleep duration and VI.

2. Sleep duration and visual function were subjectively assessed through self-reported questionnaires. If an objective assessment of sleep quality had been obtained using polysomnography or actigraphy, and vision had been tested by visual acuity chart, the results would be more robust. However, self-reported questionnaires of sleep duration and visual function are widely accepted in a number of large-scale population-based studies, and self-reported sleep duration is sufficiently consistent with objective methods, such as actigraphy $(44,45)$.

3. Self-reported sleep duration could not allow for the differentiation between total sleep time and time in bed. However, previous studies have shown that objective measures of total sleep time and time in bed were highly correlated $(7,46)$.

4. The correlation between the severity of VI and the severity of sleep difficulties was not explained. There was a lack of data about the severity of VI and the severity of sleep difficulties in the CHARLS survey. Future studies may need to address this problem.

5. The study was not adjusted for mood, medications, or exposure to artificial light at night, which may affect sleep duration $(47,48)$. They were not taken into account in previous studies $(37,48)$. However, we found the relationship was maintained throughout all three models after adjusting for sociodemographic data, lifestyle factors, and health conditions. Future studies may need to take more factors into account.

\section{CONCLUSIONS}

In summary, based on large-scale population-based data in China, both short and long sleep durations showed an association with VI. However, the cause-and-effect relationship is unclear. The relationship of VI to sleep in the elderly requires more attention because of a lack of epidemiological studies in China. A more comprehensive and integrated healthcare and rehabilitation system that addresses vision and sleep is also needed.

\section{DATA AVAILABILITY STATEMENT}

The raw data supporting the conclusions of this article will be made available by the authors, without undue reservation.

\section{AUTHOR CONTRIBUTIONS}

MS, QB, and MZ performed material preparation, data collection, and analysis. MS wrote the first draft of the manuscript and all authors commented on previous versions of the manuscript. All authors contributed to the study 
conception and design. All authors read and approved the final manuscript.

\section{FUNDING}

This research was supported by the Shanghai Natural Science Foundation (19ZR1440900), Shanghai Pujiang Program

\section{REFERENCES}

1. Flaxman SR, Bourne RRA, Resnikoff S, Ackland P, Braithwaite T, Cicinelli MV, et al. Global causes of blindness and distance vision impairment 19902020: a systematic review and meta-analysis. Lancet Glob Health. (2017) 5:e1221-34. doi: 10.1016/S2214-109X(17)30393-5

2. Tang Y, Wang X, Wang J, Huang W, Gao Y, Luo Y, et al. Prevalence and causes of visual impairment in a Chinese adult population: the Taizhou Eye study. Ophthalmology. (2015) 122:1480-8. doi: 10.1016/j.ophtha.2015.03.022

3. Wang J, Xiao LD, Wang K, Luo Y, Li X. Cognitive impairment and associated factors in rural elderly in North China. J Alzheimers Dis. (2020) 77:124153. doi: 10.3233/JAD-200404

4. Rong $\mathrm{H}$, Lai $\mathrm{X}$, Jing $\mathrm{R}$, Wang $\mathrm{X}$, Fang $\mathrm{H}$, Mahmoudi E. Association of sensory impairments with cognitive decline and depression among older adults in China. JAMA Netw Open. (2020) 3:e2014186. doi: 10.1001/jamanetworkopen.2020.14186

5. Tseng YC, Liu SH, Lou MF, Huang GS. Quality of life in older adults with sensory impairments: a systematic review. Qual Life Res. (2018) 27:195771. doi: 10.1007/s11136-018-1799-2

6. Zizi F, Jean-Louis G, Magai C, Greenidge KC, Wolintz AH, Heath-Phillip O. Sleep complaints and visual impairment among older Americans: a community-based study. J Gerontol A Biol Sci Med Sci. (2002) 57:M6914. doi: 10.1093/gerona/57.10.M691

7. Ramos AR, Wallace DM, Williams NJ, Spence DW, Pandi-Perumal SR, Zizi F, et al. Association between visual impairment and sleep duration: analysis of the 2009 National Health Interview Survey (NHIS). BMC Ophthalmol. (2014) 14:115. doi: 10.1186/1471-2415-14-115

8. Grandner MA, Drummond SP. Who are the long sleepers? Towards an understanding of the mortality relationship. Sleep Med Rev. (2007) 11:34160. doi: 10.1016/j.smrv.2007.03.010

9. Cash RE, Beverly Hery CM, Panchal AR, Bower JK. Association between sleep duration and ideal cardiovascular health among US adults, National Health and Nutrition Examination Survey, 2013-2016. Prev Chronic Dis. (2020) 17:E43. doi: 10.5888/pcd17.190424

10. Nakakubo S, Makizako H, Doi T, Tsutsumimoto K, Hotta R, Lee S, et al. Long and short sleep duration and physical frailty in community-dwelling older adults. J Nutr Health Aging. (2018) 22:1066-71. doi: 10.1007/s12603-0181116-3

11. Cappuccio FP, D'Elia L, Strazzullo P, Miller MA. Sleep duration and all-cause mortality: a systematic review and meta-analysis of prospective studies. Sleep. (2010) 33:585-92. doi: 10.1093/sleep/33. 5.585

12. Kwon S, Lee H, Lee JT, Shin MJ, Choi S, Oh H. Sleep duration and mortality in Korean adults: a population-based prospective cohort study. BMC Public Health. (2020) 20:1623. doi: 10.1186/s12889-02009720-3

13. Kwok CS, Kontopantelis E, Kuligowski G, Gray M, Muhyaldeen A, Gale CP, et al. Self-reported sleep duration and quality and cardiovascular disease and mortality: a dose-response meta-analysis. J Am Heart Assoc. (2018) 7:e008552. doi: 10.1161/JAHA.118.008552

14. Laposky AD, Van Cauter E, Diez-Roux AV. Reducing health disparities: the role of sleep deficiency and sleep disorders. Sleep Med. (2016) 18:36. doi: 10.1016/j.sleep.2015.01.007

15. Liu S, Lin Y, Liu X. Meta-analysis of association of obstructive sleep apnea with glaucoma. J Glaucoma. (2016) 25:1-7. doi: 10.1097/IJG.0000000000000357
(2019PJD047), and National Natural Science Foundation of China (81500714).

\section{SUPPLEMENTARY MATERIAL}

The Supplementary Material for this article can be found online at: https://www.frontiersin.org/articles/10.3389/fmed. 2021.778117/full\#supplementary-material
16. Liguori C, Palmieri MG, Pierantozzi M, Cesareo M, Romigi A, Izzi F, et al. Optic nerve dysfunction in obstructive sleep apnea: an electrophysiological study. Sleep. (2016) 39:19-23. doi: 10.5665/sleep.5308

17. Han KT, Nam JH, Park EC. Do sleep disorders positively correlate with dry eye syndrome? results of national claim data. Int J Environ Res Public Health. (2019). 16:878. doi: 10.3390/ijerph16050878

18. Zhao Y, Hu Y, Smith JP, Strauss J, Yang G. Cohort profile: the China Health and Retirement Longitudinal Study (CHARLS). Int J Epidemiol. (2014) 43:618. doi: 10.1093/ije/dys203

19. World Medical Association. World Medical Association Declaration of Helsinki: ethical principles for medical research involving human subjects. JAMA. (2013). 310:2191-4. doi: 10.1001/jama.2013.281053

20. von Elm E, Altman DG, Egger M, Pocock SJ, Gøtzsche PC, Vandenbroucke JP. The Strengthening the Reporting of Observational Studies in Epidemiology (STROBE) statement: guidelines for reporting observational studies. Epidemiology. (2007) 18:800-4. doi: 10.1097/EDE.0b013e3181577654

21. Shang X, Zhu Z, Wang W, He M. Associations of vision impairment and eye diseases with memory decline over 4 years in China and the United States. Am J Ophthalmol. (2021) 228:16-26. doi: 10.1016/j.ajo.2021.03.021

22. Broström A, Wahlin A, Alehagen U, Ulander M, Johansson P. Sex-specific associations between self-reported sleep duration, cardiovascular disease, hypertension, and mortality in an elderly population. J Cardiovasc Nurs. (2018) 33:422-8. doi: 10.1097/JCN.0000000000000393

23. Caceres BA, Hickey KT, Heitkemper EM, Hughes TL. An intersectional approach to examine sleep duration in sexual minority adults in the United States: findings from the Behavioral Risk Factor Surveillance System. Sleep Health. (2019) 5:621-9. doi: 10.1016/j.sleh.2019.06.006

24. Cao Z, Cheng Y, Li S, Yang H, Sun L, Gao Y, et al. Mediation of the effect of serum uric acid on the risk of developing hypertension: a population-based cohort study. J Transl Med. (2019) 17:202. doi: 10.1186/s12967-019-1953-9

25. Lei X, Sun X, Strauss J, Zhao Y, Yang G, Hu P, et al. Health outcomes and socio-economic status among the mid-aged and elderly in China: evidence from the CHARLS national baseline data. J Econ Ageing. (2014) 3:2943. doi: 10.1016/j.jeoa.2014.05.001

26. Wang Z, Li C, Yang Z, Ma J, Zou Z. Fetal and infant exposure to severe Chinese famine increases the risk of adult dyslipidemia: results from the China health and retirement longitudinal study. BMC Public Health. (2017) 17:488. doi: 10.1186/s12889-017-4421-6

27. Yuan X, Liu T, Wu L, Zou Z-Y, Li C. Validity of self-reported diabetes among middle-aged and older Chinese adults: the China Health and Retirement Longitudinal Study. BMJ Open. (2015) 5:e006633. doi: 10.1136/bmjopen-2014-006633

28. National Kidney Foundation. K/DOQI clinical practice guidelines for chronic kidney disease: evaluation, classification, and stratification. Am J Kidney Dis. (2002). 39:S1-266. doi: 10.1016/S0272-6386(02)70054-1

29. Lv S, Shen Z, Zhang H, Yu X, Chen J, Gu Y, et al. Association between exposure to the Chinese famine during early life and the risk of chronic kidney disease in adulthood. Environ Res. (2020) 184:109312. doi: 10.1016/j.envres.2020.109312

30. Ng WY, Teo BW, Tai ES, Sethi S, Lamoureux E, Tien Yin W, et al. Cystatin C, chronic kidney disease and retinopathy in adults without diabetes. Eur J Prev Cardiol. (2016) 23:1413-20. doi: 10.1177/2047487316637182

31. Li C, Lumey L. Impact of disease screening on awareness and management of hypertension and diabetes between 2011 and 2015: results from the China health and retirement longitudinal study. BMC Public Health. (2019). 19:421. doi: 10.1186/s12889-019-6753-x 
32. Rim TH, Nam JS, Choi M, Lee SC, Lee CS. Prevalence and risk factors of visual impairment and blindness in Korea: the Fourth Korea National Health and Nutrition Examination Survey in 2008-2010. Acta Ophthalmol. (2014) 92:e317-25. doi: 10.1111/aos.12355

33. Chen X, Zhou D, Shen J, Wu Y, Sun Q, Dong J, et al. Prevalence and causes of visual impairment in adults in Binhu District, Wuxi, China. Med Sci Monit. (2018) 24:317-23. doi: 10.12659/MSM.908218

34. Bourne RR, Stevens GA, White RA, Smith JL, Flaxman SR, Price H, et al. Causes of vision loss worldwide, 1990-2010: a systematic analysis. Lancet Glob Health. (2013) 1:e339-49. doi: 10.1016/S2214-109X(13)70113-X

35. Stepanski EJ. The effect of sleep fragmentation on daytime function. Sleep. (2002) 25:268-76. doi: 10.1093/sleep/25.3.268

36. Asplund R. Sleep, health and visual impairment in the elderly. Arch Gerontol Geriatr. (2000) 30:7-15. doi: 10.1016/S0167-4943(99)00045-X

37. Whinnery J, Jackson N, Rattanaumpawan P, Grandner MA. Short and long sleep duration associated with race/ethnicity, sociodemographics, and socioeconomic position. Sleep. (2014) 37:601-11. doi: 10.5665/sleep.3508

38. Moore PJ, Adler NE, Williams DR, Jackson JS. Socioeconomic status and health: the role of sleep. Psychosom Med. (2002) 64:337-44. doi: 10.1097/00006842-200203000-00018

39. Tielsch, JM. Socioeconomic status and visual impairment among urban Americans. Arch Ophthalmol. (1991). 109:63741. doi: 10.1001/archopht.1991.01080050051027

40. Pascolini D, Mariotti SP. Global estimates of visual impairment: 2010. $\mathrm{Br} \mathrm{J}$ Ophthalmol. (2012) 96:614-8. doi: 10.1136/bjophthalmol-2011-300539

41. Anujuo K, Stronks K, Snijder MB, Jean-Louis G, Ogedegbe G, Agyemang C. Ethnic differences in self-reported sleep duration in the Netherlands-the HELIUS study. Sleep Med. (2014) 15:1115-21. doi: 10.1016/j.sleep.2014.04.019

42. Jan MMS. Melatonin for the treatment of handicapped children with severe sleep disorders. Pediatr Neurol. (2000) 23:22932. doi: 10.1016/S0887-8994(00)00188-0

43. Yan SS, Wang MS, Yuan Q, Yang L, Suodi Z, Wei W. The effect of cataract surgery on salivary melatonin and sleep quality in aging people. Chronobiol Int. (2016) 33:1064-72. doi: 10.1080/07420528.2016.1197234

44. Lockley SW, Skene DJ, Arendt J. Comparison between subjective and actigraphic measurement of sleep and sleep rhythms.
J Sleep Res. (1999) 8:175-83. doi: 10.1046/j.1365-2869.1999. 00155.x

45. Hale L, Do DP. Racial differences in self-reports of sleep duration in a population-based study. Sleep. (2007) 30:1096103. doi: 10.1093/sleep/30.9.1096

46. Patel SR, Blackwell T, Ancoli-Israel S, Stone KL. Sleep characteristics of self-reported long sleepers. Sleep. (2012) 35:641-8. doi: 10.5665/sleep. 1822

47. Paksarian D, Rudolph KE, Stapp EK, Dunster GP, He J, Mennitt D, et al. Association of outdoor artificial light at night with mental disorders and sleep patterns among US adolescents. JAMA Psychiatry. (2020) 77:126675. doi: 10.1001/jamapsychiatry.2020.1935

48. Peltzer K, Phaswana-Mafuya N. Association between Visual impairment and low vision and sleep duration and quality among older adults in South Africa. Int J Environ Res Public Health. (2017). 14:811. doi: 10.3390/ijerph14070811

Conflict of Interest: The authors declare that the research was conducted in the absence of any commercial or financial relationships that could be construed as a potential conflict of interest.

The reviewer BJ declared a shared affiliation with several of the authors QB, $\mathrm{BL}, \mathrm{XS}$, and MZ to the handling editor at time of review.

Publisher's Note: All claims expressed in this article are solely those of the authors and do not necessarily represent those of their affiliated organizations, or those of the publisher, the editors and the reviewers. Any product that may be evaluated in this article, or claim that may be made by its manufacturer, is not guaranteed or endorsed by the publisher.

Copyright (c) 2021 Sun, Bo, Lu, Sun and Zhou. This is an open-access article distributed under the terms of the Creative Commons Attribution License (CC BY). The use, distribution or reproduction in other forums is permitted, provided the original author(s) and the copyright owner(s) are credited and that the original publication in this journal is cited, in accordance with accepted academic practice. No use, distribution or reproduction is permitted which does not comply with these terms. 